\title{
Ganser syndrome: examining the aetiological debate through a systematic case report review
}

\author{
Santhushi Mendis* \\ Richard E. Hodgson** \\ * Coventry and Warwickshire NHS \\ Partnership Trust, Swanswell Point, \\ Coventy \\ ** Lymebrook Mental Health Centre, \\ Talke Road, Newcastle-under-Lyme \\ UNITED KINGDOM
}

\begin{abstract}
Background and Objectives: The aetiology of the syndrome described by Ganser in 1898 has been the subject of disagreement. Proposed causes include hysteria, psychosis and stress. In addition, the presence of the syndrome in association with medical conditions raises the possibility of an organic aetiology. The authors have explored the aetiology by reviewing identified case reports published in English.

Methods: 94 case reports were obtained by searching medical databases and by conducting a hand search for possible references. This is the largest systematic review of which the authors are aware.

Results: The review indicates that the syndrome generally occurs in instances where the individual is faced with stress, especially in those with premorbid vulnerabilities. Individuals who had affective, psychotic as well as organic conditions were found to manifest the condition. However, whilst comorbid mental illness is present in some instances, the Ganser syndrome complex was found to occur commonly in the absence of other mental illnesses.

Conclusions: The absence of a comorbid mental illness in the majority of cases may suggest a specific aetiological mechanism which supports the argument that Ganser syndrome should be ranked as a distinct nosological entity along with some other rare psychiatric disorders.
\end{abstract}

Received: 31 October 2010

Revised: 23 November 2011

Accepted: 24 November 2011 


\section{Introduction}

In 1898, S.J. Ganser described three prisoners who displayed "Vorbeigehen" (approximate answers), clouding of consciousness, auditory and visual "hallucinations", and amnesia for the period during which these symptoms were manifest ${ }^{1}$. Enoch and Trethowan $^{2}$ regarded the main features of the syndrome to be

(1) approximate answers,

(2) somatic conversion symptoms,

(3) clouding of consciousness, and

(4) pseudo-hallucinations.

The tendency to give approximate answers, is regarded to be the central feature of the disorder ${ }^{3}$. Approximate answers demonstrate an astonishing deficit of knowledge even to the most basic questions, although it is apparent that the sense of the question has been understood ${ }^{4}$. As Lishman notes, approximate answers may be provided to simple addition, the naming of colours, or to simple questions pertaining to everyday matters 5 . These responses are given with serious intent, and can sometimes be interspersed with accurate answers ${ }^{5}$. Of course, the concept relies on the subjective interpretation on the part of the examiner, and it can be sometimes difficult to decide how approximate an answer should be in order to be classed as such 5 .

Since the description of the syndrome, there has been disagreement about the exact aetiology. Ganser and others such as Tsoi ${ }^{6}$ regarded the syndrome to be of an hysterical nature. However, others have disagreed. Whitlock, regarded it as a psychosis, occurring either after acute cerebral trauma or during the course of an acute psychotic illness ${ }^{7}$. Others have argued that it is a stress induced disorder which lies between malin- gering and hysteria ${ }^{8}$. Merckelbach et al, described a case where a middle aged man who presented with Ganser symptoms was in fact found to be malingering 9 . They concluded that Ganser like symptoms should be evaluated carefully preferably with tests for malingering 9 . To add to the aetiological debate, the condition has been described in association with organic conditions. For instance, Ganser symptoms have been described in frontal-temporal lobe dementia ${ }^{10}$, cerebral injury ${ }^{11}$, and in association with a space occupying lesion of the dominant hemisphere ${ }^{12}$. This, together with the fact that there is an elevated incidence of head injuries in individuals who go on to develop the syndrome ${ }^{8}$, would raise the possibility of an organic contribution in some cases.

Although S.J. Ganser described the symptoms in prisoners the condition has also been reported in the civilian population. It is thought to occur in individuals who are faced with stressful and intolerable situations ${ }^{13}$. Those who for various reasons are poorly prepared to cope with stress are thought to be the most likely to develop the symptoms ${ }^{8}$. The Ganser syndrome has also been described in cases involving litigation ${ }^{14}$.

\section{Aim}

To examine the aetiological debate by analysing case reports published in English.

\section{Method}

The following databases were searched:

- Allied and complementary medicine1985 to date.

- British Nursing Index-1994 to date. 
- Cumulative Index to Nursing and Allied Health Literature (R)-1982 to date.

- DH-DATA-1983 to date.

- Excerpta Medica Database (EMBASE) -1974 to date.

- Excerpta Medica Database (EMBASE) -1996 to date.

- King's Fund-1979 to date.

- MEDLINE-1950 to date.

- MEDLINE-1996 to date.

- PsycINFO-1806 to date.

In order to make the search as comprehensive as possible all available articles on the Ganser syndrome published in English were identified by using Medical Entry Subject Headings terms "ganser", "approximate answers" and "malingering". Hand searches and review of identified references were also carried out.

\section{Results}

59 papers were found, and altogether a total of 94 case reports were obtained. Most of the articles originated in the developed world, with the main contribution coming from the United States of America (see Appendix).

The number of cases published in a paper ranged from one to seven (see Appendix).

The year of publication ranged from 1905 to 2006 (see Appendix).

\section{Demographic characteristics}

Of the total sample, $73(77.65 \%)$ were male and $21(22.34 \%)$ were female.

Their ages ranged from 8 years to 66 years. In $9(9.6 \%)$ cases the age of the indi- vidual was not stated. In the remaining 85 cases $(90.4 \%)$ the mean age of the sample was 31.6 years. 14 individuals were aged 16 years or younger. This group represented $14.9 \%$ of the total sample.

\section{Ganser symptom complex}

The features of the syndrome considered were those cited by Enoch and Trethowan.

Although considered to be essential for the diagnosis, approximate answers were cited as occurring in only $83(88.3 \%)$ cases. It is not clear why the authors felt that those individuals who did not display approximate answers had Ganser syndrome. The figures for clouding of consciousness, somatic conversion symptoms, and hallucinations were $80(85.1 \%), 41(43.6 \%)$, and 27 $(28.7 \%)$ respectively.

Table 1

\begin{tabular}{ll}
\hline Approximate answers & $83(88.3 \%)$ \\
Clouding of consciousness & $80(85.1 \%)$ \\
Somatic conversion symptoms & $41(43.6 \%)$ \\
Hallucinations & $27(28.7 \%)$
\end{tabular}

Only $1(1 \%)$ case out of the total sample, did not exhibit any of the features described by the above authors. Only 11 cases $(11.7 \%)$ exhibited all four of the symptoms. 10 cases (10.6\%) displayed only one symptom. The number of cases which displayed 2 symptoms was $38(40.4 \%)$, whilst 34 subjects $(36.17 \%)$ displayed 3 of the above symptoms.

Table 2

\begin{tabular}{lr}
\hline $\begin{array}{l}\text { No features of Ganser syndrome as } \\
\text { described by Enoch and Trethowan }\end{array}$ & $1(1 \%)$ \\
1 symptom & $10(10.6 \%)$ \\
2 symptoms & $38(40.4 \%)$ \\
3 symptoms & $34(36.17 \%)$ \\
All 4 symptoms & $11(11.7 \%)$ \\
\hline
\end{tabular}




\section{Pre-morbid vulnerabilities}

The case reports documented a variety of difficulties prior to the onset of Ganser symptoms (see Table 3). In some cases, more than one pre-morbid vulnerability was documented. Considering the sample as a whole, 82 individuals $(87.2 \%)$ had an identified premorbid vulnerability, whilst in 12 cases $(12.8 \%)$ no premorbid vulnerability was described.

12 individuals $(12.7 \%)$ had a difficult early life and $4(4.3 \%)$ had been victims of physical or sexual abuse. $13(13.8 \%)$ had experienced scholastic difficulties.

Previous mental illness was relatively common with 39 people $(41.5 \%)$ having a previous mental illness, and $11(11.7 \%)$ having a history of dissociation. 9 individuals $(9.6 \%)$ had a history of deliberate self harm, and personality difficulties were described in $13(13.8 \%)$ cases. A history of unexplained physical symptoms was described in 8 $(8.5 \%)$ reports.

$32(34 \%)$ had a past criminal history, 13 $(13.8 \%)$ had a history of alcohol misuse, and $8(8.5 \%)$ had used illicit substances in the past.

$21(22.3 \%)$ out of the total sample, had had a cerebral injury. Of this number in 15 cases the cerebral insult had been recent, whilst in 6 individuals it had occurred in the distant past.

Table 3

\begin{tabular}{lrrr} 
Premorbid vulnerability & \multicolumn{1}{c}{ Present } & Not present & Not stated in report \\
\hline Dissociation & $11(11.7 \%)$ & $0(0 \%)$ & $83(88.3 \%)$ \\
Cerebral insult & $21(22.3 \%)$ & $2(2.1 \%)$ & $71(75.6 \%)$ \\
Deliberate self harm & $9(9.6 \%)$ & $0(0 \%)$ & $85(90.4 \%)$ \\
Sexual/physical abuse & $4(4.3 \%)$ & $0(0 \%)$ & $90(95.7 \%)$ \\
Difficult childhood & $12(12.8 \%)$ & $8(8.5 \%)$ & $74(78.7 \%)$ \\
Difficulties at school & $13(13.8 \%)$ & $9(9.6 \%)$ & $72(76.6 \%)$ \\
Alcohol abuse & $13(13.8 \%)$ & $5(5.3 \%)$ & $76(80.9 \%)$ \\
Illicit drug abuse & $8(8.5 \%)$ & $3(3.2 \%)$ & $83(88.3 \%)$ \\
Criminal history & $32(34 \%)$ & $0(0 \%)$ & $62(66 \%)$ \\
Past history of mental illness & $39(41.5 \%)$ & $12(12.8 \%)$ & $43(45.7 \%)$ \\
Personality difficulties & $13(13.8 \%)$ & $8(8.5 \%)$ & $73(77.6 \%)$ \\
History of unexplained physical symptoms & $8(8.5 \%)$ & $0(0 \%)$ & $86(91.5 \%)$ \\
Mental impairment & $5(5.3 \%)$ & $19(20.2 \%)$ & $70(74.5 \%)$ \\
\hline
\end{tabular}

It is not possible to compare the individuals with a history of mental illness with those without one due to the small sample size of the latter. However, superficially there were no obvious differences between the two groups.

\section{Context in which the symptoms arose}

27 individuals (28.7\%) who developed the symptoms were under investigation, or had been charged or arrested for a crime. Eight 
cases $(8.5 \%)$ arose in the context of compensation claims, whilst a similar number $(\mathrm{n}=8$, $8.5 \%$ ) followed accidents which did not involve litigation or compensation. 18 (19.14\%) cases of Ganser syndrome occurred in individuals who were facing domestic or occupational stressors.

Table 4

\begin{tabular}{lr}
\hline Related to a crime & $27(28.6 \%)$ \\
Compensation claim & $8(8.5 \%)$ \\
Accident not related to compensation & $8(8.5 \%)$ \\
Domestic/occupational stressor & $18(19.15 \%)$ \\
Cerebral injury & $6(6.38 \%)$ \\
Other stressors & $15(16 \%)$ \\
Context not stated & $12(12.8 \%)$ \\
\hline
\end{tabular}

\section{Mental illness}

In 1 case ( $1 \%$ of the total sample), a severe depressive disorder preceded the onset of the symptoms. In that case, the development of the Ganser syndrome is reported to have coincided with the sudden remission of the depressive illness.

In 45 cases of the total sample $(47.8 \%)$ a mental illness occurred whilst the individual displayed the Ganser symptom complex. In this group where the features of a discrete mental illness and the Ganser symptom complex occurred simultaneously, 14 had a psychotic illness, 15 had an organic mental illness, 6 had a depressive illness, 2 had a psychotic depression. 4 suffered from a stress or a grief reaction and 3 had Tourette's syndrome.

Out of the entire sample, 49 cases $(52.12 \%)$ demonstrated no features of a mental illness whilst exhibiting Ganser symptoms. Out of this cohort, 5 individuals were considered to be malingering, whilst the other 44 were not.

7 cases out of the entire sample $(7.4 \%)$, developed a mental illness after the Ganser symptoms had resolved. Out of this cohort 4 cases developed a depressive illness, 2 individuals developed mania, and 1 person developed a psychotic episode.

Table 5

\begin{tabular}{ll}
\hline Mental illness preceding the syndrome & $1(1 \%$ of the total sample $)$ \\
Severe depression & 1 \\
\hline Mental illness coexisting with the syndrome & 45 (47.8\% of the total sample) \\
Psychosis/schizophrenia & 14 \\
Depression & 6 \\
Psychotic depression & 2 \\
Organic mental illness & 15 \\
Stress/grief reaction & 4 \\
Tourettes syndrome & 3 \\
Diagnosis unsure & 1 \\
\hline No mental illness occurring with the syndrome & $49(52.12 \%$ of the total sample $)$ \\
Malingering & 5 \\
Not malingering & 44 \\
\hline Mental illness occurring after the syndrome had resolved & $7(7.4 \%$ of the total sample $)$ \\
Mania & 2 \\
Depression & 4 \\
Psychosis & 1 \\
\hline
\end{tabular}




\section{Amnesia for the event}

It was not documented in 61 cases $(64.9 \%)$ as to whether the individual had amnesia for the symptoms or not.

In the remaining 33 cases it was documented whether or not amnesia for the event was present. Within this group, 25 had total amnesia for the symptoms $(75.75 \%)$; in 3 cases the amnesia was partial, and in 5 cases there was no amnesia present as the individuals had total recollection of their symptoms.

\section{Children with Ganser's syndrome}

14 individuals in the sample were 16 years old or younger. There was no history of physical or sexual abuse in any of the cases. In 4 cases $(28.6 \%)$ there was a history of previous head injury. $6(42.9 \%)$ children were reported to have experienced problems at school. $3(21.4 \%)$ had a forensic history. 6 individuals had a diagnosis of a mental illness, with 3 individuals described as having Tourette's syndrome, 1 suffering from nocturnal enuresis, and 2 suffering from psychotic illnesses.

\section{Discussion}

As far as we are aware this is the largest systematic review of all the case reports published so far. However, our review is subject to bias as only case reports published in the English language have been abstracted. Articles on Ganser syndrome have been published in the German language. For example, Wirtz et al. (2008) describe a patient with Ganser's syndrome after a large left-hemispheric middle cerebral artery infarct. They reported that detailed neuropsychological examination revealed atypical lateralisation of cognitive functions. They postulated that approximate answers was possibly associated with frontal-executive cerebral dysfunction and concluded that brain injury was a common aetiological factor ${ }^{15}$.

As the majority of reports are of subjects from the developed world, the clinical phenomenology may be different in other settings. As there is no agreed standard for case reporting it may mean that relevant information was not described. Case reports also do not allow an estimate of the incidence or prevalence of the disorder. Moreover, as the duration of symptoms was so poorly recorded, little information could be gleaned on the natural history of the disorder.

Only $11.7 \%$ of cases displayed all four of the central features described by Enoch and Trethowan. This proves that the presence of all four features is the exception rather than the rule.

Both ICD-10 ${ }^{16}$ and DSM-IV ${ }^{17}$ do not specify any diagnostic criteria, apart from mentioning the presence of approximate answers as being required for the diagnosis. Many consider the giving of approximate answers to be the central feature of this disorder. However, this feature was described in only $88.3 \%$ of cases. This raises the question as to whether the remainder actually involved genuine cases of Ganser syndrome. It may mean that these individuals were misdiagnosed as having the disorder, when in fact they were suffering from other mental disorders. For instance, the confabulation and amnesia associated with Wernicke-Korsakoff's syndrome could be mistaken for Ganser's syndrome. Moreover, the fact that 5 individuals who were initially thought to be displaying Ganser syndrome were later found to be fabricating their symptoms, sug- 
gests that malingering is an important differential diagnosis which needs to be ruled out at the outset.

Although Ganser described the syndrome in prisoners our review showed that only $28.6 \%$ of cases involved those who were accused of committing an offence. In fact, the majority of cases involved the civilian population. Moreover, the fact that some cases occurred in those who were aged 16 years or less, indicates that the syndrome is not confined to adults.

Although the context in which the symptoms arose was not mentioned in 12 cases (12.8\%) the remaining 82 individuals $(87.2 \%)$ which comprised the majority of the sample had been exposed to a stressor of some description. In 15 instances (16\% of the total sample) the individuals had experienced a cerebral insult. Whilst suffering from a cerebral insult is stressful in itself, the resulting change to the brain's architecture could impair the individual's capacity to cope with stress. Both these findings could allow one to hypothesise that stress plays a significant aetiological role in the disorder.

49 cases $(52.12 \%$ of the total sample) who had Ganser's syndrome did not show features of a co-morbid mental disorder. This proves that the syndrome can occur in apparently healthy individuals who have been facing a stressor of some description. However, a significant percentage $(45.7 \% \mathrm{n}$ $=43$ ) developed the symptoms while experiencing a mental disorder. It cannot be stated with certainty whether the manifestations of the mental disorder were mistaken as Ganser syndrome, or whether the two disorders occurred independently. Alternatively, it could be argued that having a mental disorder could lead to the emergence of Ganser symptoms by reducing the individual's capability to cope with stress.
Heron et al. ${ }^{8}$ stated that individuals who have pre-morbid vulnerabilities are the most vulnerable to developing the syndrome. The fact that pre-morbid vulnerabilities were described in the majority of case reports $(87.2 \%)$, lends weight to this hypothesis.

F.A. Whitlock regarded the syndrome as a psychotic one ${ }^{7}$. This view can be discounted based on the finding that a only minority of cases were felt to be exhibiting psychotic symptoms along with the Ganser symptoms. If Whitlocks's view was true, then every single case would have been considered to be suffering from a psychotic illness by the respective author. However, this was not the case.

Moreover, it cannot be argued that it is purely an organic disorder, as not all cases had an underlying organic illness, whilst exhibiting the symptoms. In fact, only 15 cases (15.9\% of the total sample) did. However, an organic contribution is suggested by the fact that a significant proportion of the sample $(22.3 \%)$ had suffered from a cerebral insult at some point.

In support of the view that the syndrome has features of an hysterical disorder is the finding that out of the cases where it was documented whether there was amnesia for the event or not, the majority of cases $(75.75 \%)$ had no recollection of their symptoms. It is interesting to note that DSM-1V categorises the syndrome as a dissociative one as well.

What is apparent is that the syndrome generally occurs in instances where the individual is faced with stress, and that those whose ability to cope under such situations is compromised due to pre-morbid vulnerabilities, are particularly at risk.

However, it is not possible to state with certainty about the exact aetiology of the disorder. This is because individuals suffer- 
ing from affective, psychotic, as well as organic disorders have exhibited the syndrome. Moreover, there is a possibility that the syndrome may have features of an hysterical disorder as well. There is therefore a difficulty with the nosological status of the syndrome. This has led some to argue that it should be ranked as a distinct nosological entity along with other rare and exotic psychiatric disorders ${ }^{5}$. The treatment offered varied as well. In some cases no treatment was offered. In instances where treatment was used, it was variable, and included psychotherapy, electro convulsive therapy and antipsychotic medication.
In conclusion, this review shows that the syndrome generally occurs in instances where the individual is faced with stress and has a premorbid vulnerability which may diminish their capacity to cope in such situations. Patients presenting with all the originally described features are a rarity. Whilst comorbid mental illness is present in some cases the Ganser syndrome cluster does occur commonly in the absence of other mental illnesses which suggests specific aetiological mechanisms. Whilst case reports offer little information on epidemiology the fact there are no specific questions on $\mathrm{SCAN}^{18}$ and $\mathrm{SCID}^{19}$ may indicate the rarity of the disorder.

\section{Appendix 1}

Number of case reports published by country

\begin{tabular}{lr}
\hline United States of America & $41(43.6 \%)$ \\
United Kingdom and Ireland & $14(14.9 \%)$ \\
Rest of Europe & $13(13.8 \%)$ \\
Australia and New Zealand & $8(8.5 \%)$ \\
Rest of the world & $18(19.1 \%)$
\end{tabular}

Number of case reports in each paper

\begin{tabular}{lr}
\hline 1 case & $47(79.6 \%)$ \\
2 cases & $3(5.1 \%)$ \\
3 cases & $3(5.1 \%)$ \\
4 cases & $1(1.7 \%)$ \\
5 cases & $3(5.1 \%)$ \\
6 cases & $1(1.7 \%)$ \\
7 cases & $1(1.7 \%)$
\end{tabular}

Year of publication

\begin{tabular}{lc}
\hline $1900-1910$ & $4(6.8 \%)$ \\
$1910-1920$ & $0(0 \%)$ \\
$1920-1930$ & $0(0 \%)$ \\
$1930-1940$ & $0(0 \%)$ \\
$1940-1950$ & $1(1.7 \%)$ \\
$1950-1960$ & $4(6.8 \%)$ \\
$1960-1970$ & $5(8.5 \%)$ \\
$1970-1980$ & $9(15.3 \%)$ \\
$1980-1990$ & $15(25.4 \%)$ \\
$1990-2000$ & $10(16.9 \%)$ \\
$2000-2010$ & $11(18.6 \%)$ \\
\hline
\end{tabular}




\section{Appendix 2. Bibliography}

1. Apter A, Ratzoni G, Iancu J, Weizman R, Tyano S. The Ganser syndrome in two adolescent brothers. J Am Acad Child Adolesc Psychiatry 1993; 32(3): 582-584.

2. Miller P, Bramble D, Buxton N. Case study: Ganser syndrome in children and adolescents. J Am Acad Child Adolesc Psychiatry 1997; 36(1): 112-115.

3. Haddad PM. Ganser syndrome followed my major depressive episode. Br J Psychiatry 1993; 162: 251-253.

4. Adler R, Touyz S. Ganser syndrome in a 10 year old boy - an 8 year follow up. Aust N Z J Psychiatry 1989; 23: 124-126.

5. Carney MW, Chary TK, Robotis P, Childs A. Ganser syndrome and its management. Br J Psychiatry 1987; 151: 697-700.

6. Feinstein A, Hattersley A. Ganser symptoms, dissociation, and dysprosody. J Nerv Ment Dis 1988; 176(11): 692-693.

7. Dabholkar PD. Ganser syndrome. A case report and discussion. Br J Psychiatry 1987; 151: 256-258.

8. Drob SL, Meehan K. The diagnosis of Ganser syndrome in the practice of forensic psychology. Am J Forensic Psychol 2000; 18(3): 37-62.

9. Lee BH, Koenig T. A case of Ganser syndrome: organic or hysterical? Gen Hosp Psychiatry 2001; 23: 230-234.

10. Andersen HS, Sestoft D, Lillebaek T. Ganser syndrome after solitary confinement in prison: A short review and case report. Nord J Psychiatry 2001; 55: 199-201.

11. Goldin S, MacDonald JE. The Ganser state. J Ment Sci 1955; 101: 267-280.

12. Lieberman AA. The Ganser syndrome in psychoses. J Nerv Ment Dis 1954; 120: 10-16.

13. Lauerma H, Hypen K. Ganser syndrome, organic dementia and hypnosis- A case study. J Trauma Dissociation 2004; 5(3): 93-99.

14. Kerbeshian J, Burd L. A second visually impaired, mentally retarded male with pervasive developmental disorder, Tourette disorder and Ganser's syndrome: Diagnostic classification and treatment. Int J Psychiatry Med 19861987; 16(1): 67-75.

15. Arya DK. Resolution of episode of major depressive disorder with emergence of Ganser syndrome symptoms. Ir J Psychol Med 1997; 14(1): 35-37.

16. Bustamante JP, Ford CV. Ganser's syndrome. Psychiatr Opin 1977; 14(5): 39-41.
17. Refaat R, Firth DA, Robertson MM. Uncomplicated Gilles de la Tourette syndrome and probable Ganser syndrome: A case report and review of the literature. Eur Child Adolesc Psychiatry 2002; 11(5): 234-239.

18. Nardi TJ, Di Scipio WJ. The Ganser syndrome in an adolescent Hispanic-Black female. Am J Psychiatry 1977; 134(4): 453-454.

19. Rieger W, Billings CK. Ganser's syndrome associated with litigation. Compr Psychiatry 1978; 19(4): 371-375.

20. Schneider R, Klosinski G. Travellers in five worlds: adolescents with Ganser's syndrome. Acta Paedopsychiatr 1989; 52(5): 150-155.

21. Cocores JA, Santa WG, Patel MD. The Ganser syndrome: Evidence suggesting its clasification as a dissociative disorder. Int J Psychiatry Med 1984; 14(1): 47-56.

22. Ladowsky-Brooks RL, Fischer CE. Ganser symptoms in a case of frontal-temporal lobe dementia: Is there a common neural substrate? J Clin Exp Neuropsychol 2003; 25(6): 761-768.

23. Adler R. Pseudodementia or Ganser syndrome in a ten year old boy. Aust N Z J Psychiatry 1981; 15: 339-342.

24. Snyder SL, Buchsbaum MS, Krishna RC. Unusual visual symptoms and Ganser-like state due to cerebral injury: a case study using (18)F-deoxyglucose positron emission tomography. Behav Neurol 1998; 11(1): 51-54.

25. Hampel H, Berger C, Muller N. A case of Ganser's state presenting as a dementia syndrome. Psychopathology 1996; 29: 236-241.

26. May RH, Voegele GE, Paolino AF. The Ganser syndrome: A report of three cases. J Nerv Ment Dis 1960; 130: 331-339.

27. Weiner H, Braiman A. The Ganser syndrome: A review and addition of some unusual cases. Am J Psychiatry 1955; 111(10): 767-773.

28. Jiang H, Feng Y. Ganser syndrome-An unusual case report and literature review. Chin Med J 1988; 41: 85-88.

29. Burd L, Kerbeshian J. Tourette syndrome, atypical pervasive developmental disorder and Ganser syndrome in a 15-year-old, visually impaired, mentally retatarded boy. Can J Psychiatry 1985; 30: 74-76.

30. Deibler MX, Hacker C, Rough J, Darby J, Lamdan RM. Ganser's syndrome in a man with AIDS. Psychosomatics 2003; 44(4): 342-345.

31. Dwyer J, Reid S. Ganser's syndrome. Lancet 2004; 364: 471-473.

32. Ingraham MR, Moriarty DM. A contribution to the understanding of the Ganser syndrome. Compr Psychiatry 1967; 8(1): 35-44. 
33. Latcham R, White A, Sims A. Ganser syndrome: The aetiological argument. J Neurol Neurosurg Psychiatry 1978; 41(9): 851-854.

34. Margetts E. "Ganser syndrome" in a native African criminal. East Afr Med J 1960; 37: 32-36.

35. Steinhart MJ. Ganser state: A case of hysterical pseudodementia. Gen Hosp Psychiatry 1980; 2(3): 226-228.

36. Laing RD. An instance of the Ganser syndrome. J R Army Med Corps 1953; 99(4): 169-172.

37. Frost HP. Hysterical insanity. Report of a case presenting Ganser's symptom-complex. Am J Insanity 1907; 63: 301-308.

38. Doongaji DR, Apte JS, Bhat R. Ganser state (syndrome). An unusual presentation of a space occupying lesion of the dominant hemisphere. Neurol India 1975; 23(3): 143-148.

39. Heron EA, Kritchevsky M, Delis DC. Neuropsychological presentation of Ganser symptoms. J Clin Exp Neuropsychol 1991; 13(5): 652-666.

40. Stern ES, Whiles WH. Three Ganser states and Hamlet. J Ment Sci 1942; 88: 134-141.

41. McEvoy J, Campbell T. Ganser-like signs in carbon monoxide encephalopathy. Am J Psychiatry 1977; 134(12): 1448-1449.

42. Grieger TA, Clayton AH. A possible association of Ganser's syndrome and major depression. J Clin Psychiatry 1990; 15(10): 437.

43. Townsend TI. The Ganser symptom and symptomcomplex. Report of five cases. Am J Insanity 1910; 66: 631-647.

44. Tsoi WF. The Ganser syndrome in Singapore: A report on ten cases. Br J Psychiatry 1973; 123: 567-572.

45. Diller T, Wright GJ. A study of hysterical insanity with an especial consideration of Ganser's symptom-complex-Report of eight cases. J Nerv Ment Dis 1909; 36: 25-54.

46. Mathura S, Rastogi CK, Singh YD, Singh RN, Mathur GP. Ganser's syndrome: A rare psychiatric disorder. Indian Pediatr 1976; 13(12): 947-948.

47. Nyrio J, Iranyi C. A contribution to the interpretation of Ganser symptoms. Psychiatr Neurol 1965; 150(2): 65-73.

48. Whitlock FA. The Ganser syndrome. Br J Psychiatry 1967; 113: 19-29.

49. Cocores JA, Schlesinger LB, Gold MS. A review oft he EEG literature on Ganser's syndrome. Int J Psychiatry Med 1986; 16(1): 59-65.
50. Weller MPI. Hysterical behaviour in patriarchal communities. Four cases, one with Ganser like symptoms. Br J Psychiatry 1988; 152: 687-695.

51. Dalfen AK, Feinstein A. Head injury, dissociation and the Ganser syndrome. Brain Inj 2000; 14(12): 11011105 .

52. Peszke MA, Levin GA. The Ganser syndrome: A diagnostic and etiological enigma. Conn Med 1987; 51(2): $79-83$.

53. Mahadevappa H. Ganser syndrome: A case report. J Clin Psychiatry 1990; 51(4): 167.

54. Williams K. Ganser syndrome-100 years on. Ir J Psych Med 1999; 16(3):115-117.

55. Merckelbach H, Peters M, Jelicic M, Brands I, Smeets T. Detecting malingering of Ganser-lime symptoms with tests: A case study. Psychiatry Clin Neurosci 2006; 60(5): 636-638

56. Ruggles AH. Observations on Ganser's symptom. Am J Insanity 1905; 62: 307-312.

57. Allen DF, Postel J, Berrios GE. The Ganser syndrome. In: Berrios GE, Hodges JR, editors. Memory disorders in psychiatric practice. New York: Cambridge University Press; 2000. p. 443-455.

58. Cosgray RE, Fawley RW. Could it be Ganser's syndrome? Arch Psychiatr Nurs 1989; 3(4): 241-245.

59. Singh R. Experimental analysis of Ganser syndrome. Indian J Clin Psychol 1977; 4: 19-23.

\section{References}

1. Ganser SJ. Veber einen eigenartigan hysterischen Daminszustand. Arch Psychiatr Nervenkr 1898; 30: 633-640.

2. Enoch MD, Trethowan WH. The Ganser syndrome. In: Uncommon psychiatric syndromes. Bristol: John Wright; 1979; p. 50-62.

3. Weiner H, Braiman A. The Ganser Syndrome: a review and addition of some unusual cases. Am J Psychiatry 1955: 111(10): 767-773.

4. Apter A, Ratzoni G, Iancu I, Weizman R, Tyano S. The Ganser syndrome in two adolescent brothers. J Am Acad Child Adolescent Psychiatry 1993; 32(3): 582-584.

5. Lishman WA. Organic Psychiatry. The Psychological consequences of cerebral disorder, $3^{\text {rd }}$ edition. Oxford: Blackwell Science; 1998. 
6. Tsoi WF. The Ganser Syndrome in Singapore: A report on ten cases. Br J Psychiatry 1973; 123: 567-572.

7. Whitlock FA. The Ganser syndrome. Br J Psychiatry 1967; 113: 19-29.

8. Heron EA, Kritchevsky M, Delis DC. Neuropsychological presentation of Ganser symptoms. J Clin Exp Neuropsychol 1991; 13(5): 652-666.

9. Merckelbach H, Peters M, Jelicic M, Brands I, Smeet T. Detecting malingering of Ganser-like symptoms with tests: A case study. Psychiatry Clin Neurosci 2006; 60: 636-638.

10. Ladowsky-Brooks RL, Fischer CE. Ganser symptoms in a case of frontal-temporal lobe dementia: is there a common neural substrate? J Clin Exp Neuropsychol 2003; 25(6): 761-768.

11. Snyder SL, Buchsbaum MS, Krishna RC. Unusual visual symptoms and Ganser-like state due to cerebral injury: a case study using (18)F-deoxyglucose positron emission tomography. Behavioural Neurology. 1998:11; 51-54.

12. Doongaji DR, Apte JS, Bhat R. Ganser State (Syndrome): An unusual presentation of a space occupying lesion of the dominant hemisphere. Neurol India 1975; 23(3): 143-148.

13. Andersen HS, Sestoft D, Lillebaek T. Ganser syndrome after solitary confinement in prison: A short review and a case report. Nord J Psychiatry 2001; 55: 199-201.

14. Reiger W, Billings CK. Ganser's syndrome associated with litigation. Comp Psychiatry 1978; 19(4): 371-375.
15. Wirtz G, Baas U, Hofer H, Nyffeler T, Seifritz E, Müri R, et al. Psychopathology of Ganser syndrome. Literature review and case discussion. Der Nervenartz 2008: 79(5): 543-557.

16. World Health Organisation. The ICD-10 Classification of Mental and Behavioural Disorders: clinical descriptions and diagnostic guidelines. Geneva: World Health Organization; 1993.

17. American Psychiatric Association. Diagnostic and Statistical Manual of Mental Disorders, $4^{\text {th }}$ edition. Washington, D.C.: American Psychiatric Association; 2000.

18. World Health Organization. Schedules for Clinical Assessment in Neuropsychiatry (SCAN). Geneva: World Health Organization; 1994.

19. First MB, Spitzer RL, Gibbon M, Williams JBW. User's Guide for the Structured Clinical Interview for DSM-IV Axis I Disorders - Clinician version (SCID-CV). Washington, D.C.: American Psychiatric Press; 1997.

Author for correspondence:

Dr. Richard E. Hodgson

Consultant Psychiatrist

Lymebrook Mental Health Centre

Talke Road

Newcastle-under-Lyme, ST5 7TL

United Kingdom

E-mail: RichardE.Hodgson@ northstaffs.nhs.uk 